\title{
DESIGNING PHOTONIC BANDGAP FIBERS FOR PARTICLE ACCELERATION*
}

\author{
Robert J. Noble", Eric R. Colby, Benjamin Cowan, Christopher M. Sears, Robert H. Siemann, \\ James E. Spencer, SLAC, Stanford University, Stanford, CA 94309 USA
}

\begin{abstract}
Photonic bandgap (PBG) fibers with hollow core defects have been suggested for use as laser driven accelerator structures. The modes of a photonic crystal fiber lie in a set of allowed bands. A fiber with a central vacuum defect can support so-called defect modes with frequencies in the bandgap and electromagnetic fields confined spatially near the defect. A defect mode suitable for relativistic particle acceleration must have a longitudinal electric field in the central defect and a phase velocity at the speed of light (SOL). We explore the design of the defect geometry to support well confined accelerating modes in such PBG fibers. The dispersion diagram of an accelerating mode must cross the SOL line, and such modes form a special class of defect modes known as surface modes, which are lattice modes of the original PBG crystal that have been perturbed into the bandgap. The details of the surface boundary separating the defect from the surrounding PBG matrix are found to be the critical ingredients for optimizing the accelerator mode properties.
\end{abstract}

\section{INTRODUCTION}

Photonic crystals have been suggested for use as laserdriven particle accelerator structures [1]. A photonic crystal is a dielectric structure arranged in a periodic geometry. Like a crystalline solid with its electronic band structure, the modes of a photonic crystal lie in a set of allowed photonic bands. A photonic crystal will similarly exhibit one or more photonic band gaps, with the frequencies in the gap corresponding to modes unable to propagate in the crystal. Defect modes can be obtained by introducing a defect into the photonic crystal lattice. Since frequencies in the band gap are forbidden to propagate in the crystal, these modes are spatially confined to the defect. A longitudinal defect in a transversely periodic crystal can act as an optical waveguide.

Hollow-core photonic band gap (PBG) fibers originally attracted great interest in the telecom industry due to their unique advantage over conventional solid core fibers, namely, very low propagation loss for modes confined to the core. The geometry of this structure is a hexagonal array of hollow cylinders in a silica matrix. The central defect is typically a cylinder of larger radius than the holes that make up the surrounding matrix. The structure is drawn as a waveguide along which the light propagates.

The defect modes in hollow-core PBG fibers have become recognized as belonging to two classes: "core modes" with Poynting flux almost entirely contained

\footnotetext{
*Supported by U.S. Dept. of Energy contract DE-AC02-76SF00515

\#noble@slac.stanford.edu
}

within the core and "surface modes" which are localized at the boundary separating the defect and matrix [2], [3]. Core modes are distinguished by the fact that their dispersion relation never crosses the speed of light (SOL) line. Their effective index, $\mathrm{n}_{\mathrm{eff}}$, is always less than one, and their phase velocity, $\mathrm{c} / \mathrm{n}_{\text {eff }}$, is greater than the speed of light. An optical communication mode is an example of a core defect mode which has a transversely polarized, dipole-like field pattern. The dispersion relation of a surface mode on the other hand can cross the light line, exhibiting an effective index that can be greater than, equal to, or less than one. Surface modes generally occur whenever a periodic lattice is terminated at a boundary. Surface modes are in fact lattice modes with frequencies that have been sufficiently perturbed by the defect to lie in the band gap and can propagate along the defect.

A defect mode suitable for relativistic particle acceleration must have a longitudinal electric field in the central defect and a phase velocity at the speed of light. The dispersion relation of an accelerating mode must cross the SOL line, and consequently must be a surface mode. The recognition of accelerating modes as surface modes implies that the details of the surface boundary separating the defect from the surrounding matrix are the critical ingredients for optimizing the accelerator mode properties.

\section{PBG FIBER STRUCTURES}

The fibers in which we have investigated accelerating modes are the hexagonal fiber introduced by X.E. Lin [1], as shown in Figure 1, and a circular-hole model approximating the HC-1550-02 fiber of Crystal Fibre A/S [4], shown in Figure 2. In both figures a plot of the longitudinal electric field intensity of the accelerating mode is overlaid. The modes were calculated using the CUDOS software of the University of Sydney [5]. Note the large field intensities in the silica regions surrounding the defects, characteristic of surface modes.

The Lin fiber in Figure 1 is defined by four constants: the lattice transverse hole spacing a, which simply scales the wavelengths of the fiber modes, the ratio of hole radius to lattice spacing r/a, the ratio of central defect radius to lattice spacing $\mathrm{R} / \mathrm{a}$, and the relative permittivity of the dielectric matrix $\varepsilon_{\mathrm{r}}$. For this fiber, the parameters are $\mathrm{r} / \mathrm{a}=0.35, \mathrm{R} / \mathrm{a}=0.52$, and $\varepsilon_{\mathrm{r}}=2.13$ for silica. The lattice spacing was chosen as $1.3 \mu \mathrm{m}$ in Figure 1 to yield an SOL accelerating mode at $1 \mu \mathrm{m}$. The bandgap diagram for the perfect lattice is shown in Figure 3 as calculated with R-Soft BandSolve [6]. The band diagram is determined only by the ratio r/a and the matrix permittivity. The Lin fiber is about half silica in volume, 
which results in the band diagram consisting of several well separated gaps at relatively small values of $\omega \mathrm{a} / \mathrm{c}$. The SOL accelerating mode occurs at a normalized frequency of $\omega \mathrm{a} / \mathrm{c}=\mathrm{k}_{0} \mathrm{a}=8.2$ and resides in the second gap from the bottom (green) in Figure 3 where the SOL line crosses it.

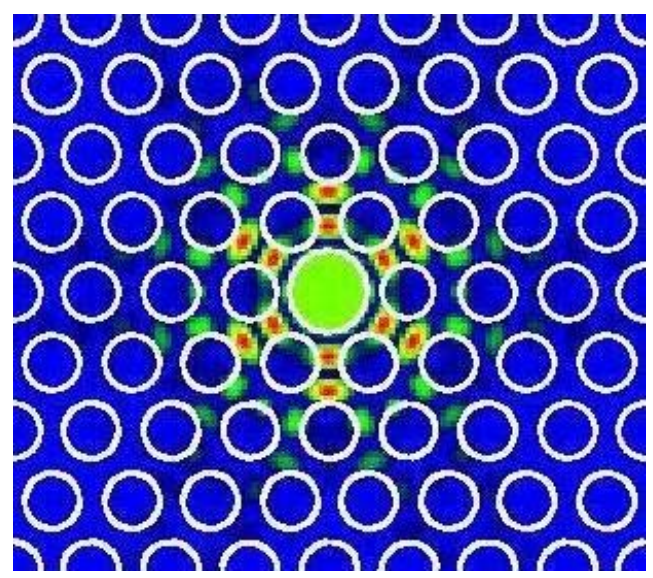

Figure 1: Accelerating field intensity of a $1 \mu \mathrm{m}$ mode in a Lin fiber as calculated with CUDOS. The longitudinal direction is out of the page. The color scale is relative with red being the highest field and blue the lowest.

The band gap diagram of the HC-1550-02 model fiber is shown in Figure 4. This fiber is only about $10 \%$ silica by volume. This shifts the band gaps to higher frequency compared to the Lin fiber and narrows all but the lowest gap. The band diagram is dominated by the lowest gap (blue) crossing the SOL line at about $\omega \mathrm{a} / \mathrm{c}=14$.

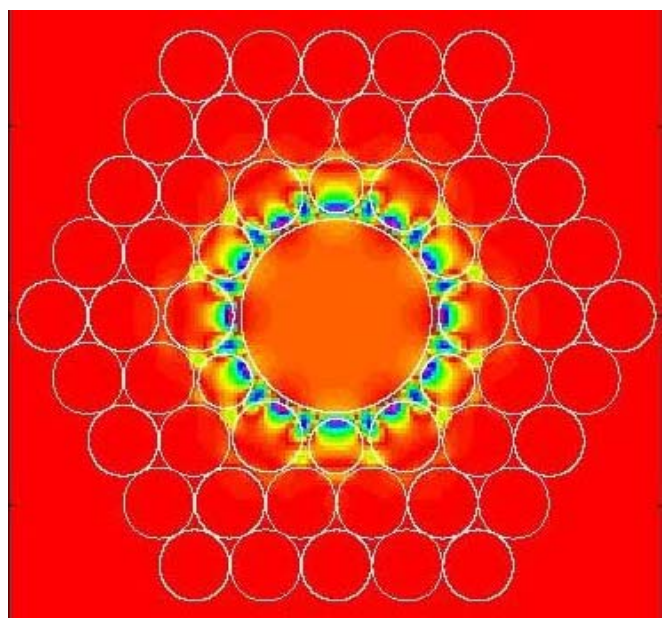

Figure 2: Accelerating field intensity of a $1.89 \mu \mathrm{m}$ mode in a circular-hole model of the HC-1550-02 fiber as calculated with CUDOS. To accent the weak fields in the core, an inverted color scale is used with blue being the highest field and red the lowest.

The dimensions for the circular-hole model of the HC1550-02 fiber were chosen to place the bandgap near 1.7 $\mu \mathrm{m}(\mathrm{r}=1.88 \mu \mathrm{m}, \mathrm{a}=3.8 \mu \mathrm{m})$. The defect hole radius is $\mathrm{R}=5.1 \mu \mathrm{m}(\mathrm{R} / \mathrm{a}=1.34)$. The first layers of holes surrounding the central hole differ in radius from the matrix, being alternately 1.45 and $1.87 \mu \mathrm{m}$, to roughly match the manufacturer's description. This is an example of a multi-layer defect. CUDOS finds a large number of defect modes in this fiber. The mode in Figure 2 is the lowest frequency accelerating mode identified.

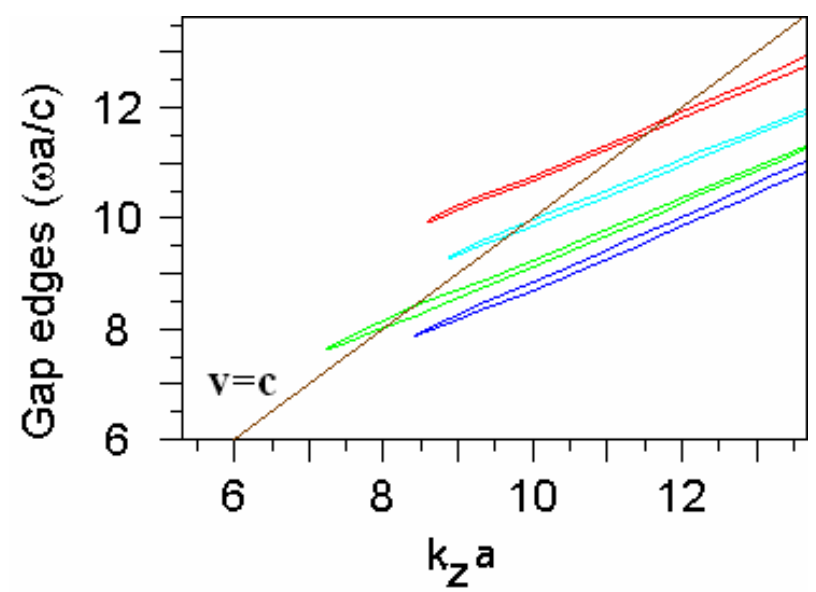

Figure 3: Band gap diagram with SOL line indicated for the Lin fiber as calculated with R-Soft BandSolve.

The HC-1550-02 fiber was designed to support coretype defect modes for telecom. Surface modes which can mode-mix or compete for power are undesirable for telecom. The number of core modes is estimated from the formula $N_{c} \approx\left(k_{0} a\right)^{2}(R / a)^{2} \Delta k / k_{0}$, where $\Delta k$ is the gap width [3]. For our HC-1550-02 model, $\mathrm{N}_{\mathrm{c}} \approx 30$, while for the Lin fiber, it is only 0.3 , indicating that the latter will support surface modes but not core modes. Competing core modes are not desirable for an accelerator fiber.

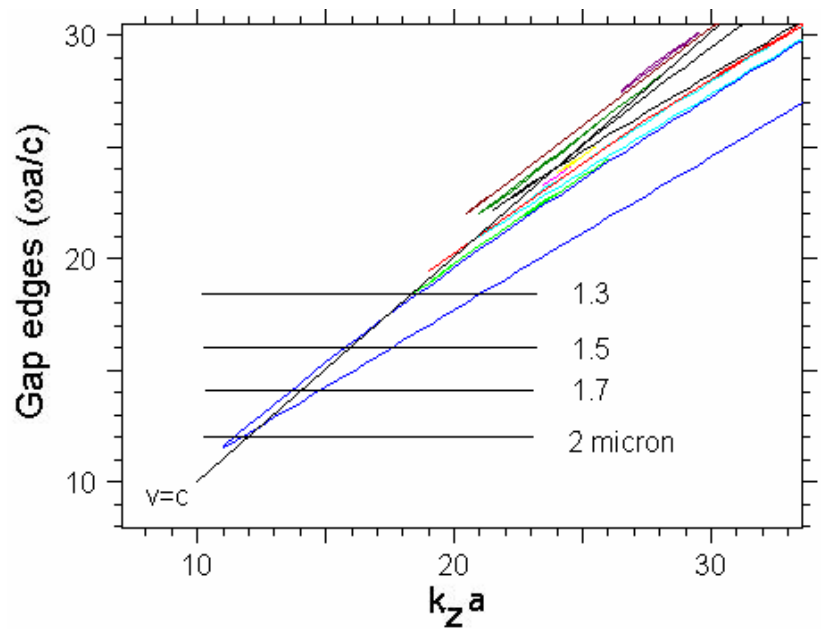

Figure 4: Band gap diagram for the circular-hole model of the HC-1550-02 fiber from R-Soft BandSolve. For reference, some free-space wavelengths are shown for a lattice hole spacing of $3.8 \mu \mathrm{m}$.

\section{Defect Geometry Variations}

A comparison of Figures 1 and 2 shows significant differences in the field patterns for these two fiber defects. The fields within the defect and at the boundary are sensitive to the interface geometry. For the Lin fiber, the 
ratio of accelerating field to the maximum field in the matrix, $E_{a c c} / E_{\max }$, is 0.48 while for the HC-1550-02 fiber this ratio is 0.066 . This difference is also reflected in the characteristic impedances for these two modes, $\mathrm{Z}_{\mathrm{c}}=\mathrm{E}_{\mathrm{acc}}{ }^{2}$ $\lambda^{2} / P_{z}$, where $\lambda$ is the mode wavelength and $P_{z}$ is the power. The impedances are $19.5 \Omega$ and $0.25 \Omega$ for the Lin and $\mathrm{HC}-1550-02$ fibers, respectively.

We have made a preliminary study of modifying the accelerating modes in these fibers by changing the defect geometry. Because these are surface modes, increasing the defect radius will perturb the accelerating mode to a higher frequency, moving it up in the band gap. For the Lin fiber, we find that the mode's wavelength shift is $\mathrm{d} \lambda / \mathrm{dR} \approx-0.1$. Conversely by reducing the defect radius, the mode moves down in the gap. For a $14 \%$ radius reduction, the mode can be shifted down from the gap center to the allowed lattice band with increased imaginary index, $\operatorname{Im}(\mathrm{n})$, which indicates less confinement.

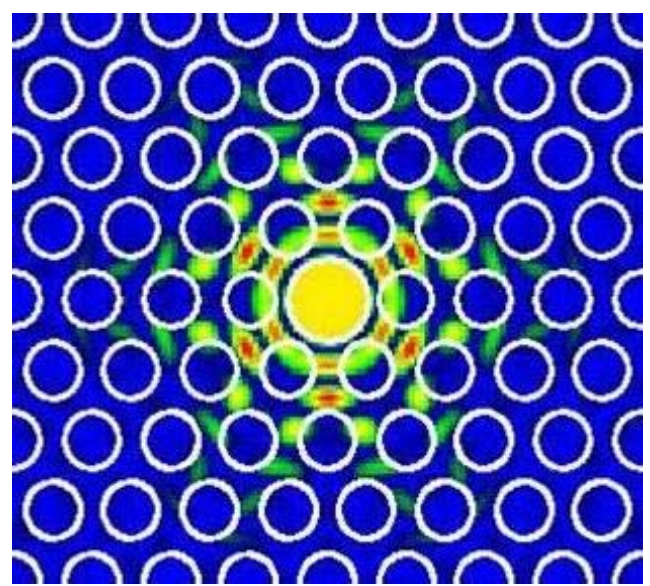

Figure 5: Increased accelerating field for a $1 \mu \mathrm{m}$ mode in a modified Lin fiber.

For the Lin fiber in Figure 1, the ratio of accelerating field to the maximum $\mathrm{E}_{\mathrm{z}}$ field in the matrix is $\mathrm{E}_{\mathrm{acc}} / \mathrm{E}_{\mathrm{z}, \max }=$ 0.62. A high $E_{z}$ in the silica is not useful for acceleration. A means of increasing the core field is suggested by the six high-field points in the silica surrounding the defect. We filled the six holes around the defect with material of permittivity $\varepsilon_{\mathrm{r}}=1.5$ and reduced their radius by $5 \%$. As shown in Figure 5, this smoothes and concentrates the spatial field pattern closer to the core, increasing the ratio to $\mathrm{E}_{\mathrm{acc}} / \mathrm{E}_{\mathrm{z}, \max }=0.78$. In this modified fiber $\mathrm{E}_{\mathrm{acc}} / \mathrm{E}_{\max }=$ 0.47 . The modification shifts $25 \%$ more longitudinal field from the silica into the core, but the ratio $\mathrm{E}_{\mathrm{acc}} / \mathrm{E}_{\max }$ remains about the same as in the original fiber. For this model, $\operatorname{Im}(\mathrm{n})$ increases from $2 \times 10^{-4}$ to $9 \times 10^{-4}$. This is due to a small increase in the fields beyond the second layer of holes, causing added diffraction. We find that $\operatorname{Im}(n)$ can be reduced by an order of magnitude for every two layers of matrix holes added. It can also be reduced by increasing the radii of holes in the second layer out from the core. Work is in progress to determine which geometry features have the strongest influence on the ratio $\mathrm{E}_{\mathrm{acc}} / \mathrm{E}_{\max }$ but maintain the improved ratio in $\mathrm{E}_{\mathrm{acc}} / \mathrm{E}_{\mathrm{z}, \max }$.
The accelerating mode in the $\mathrm{HC}-1550-02$ fiber is similarly sensitive to geometry changes. The large central defect is advantageous for the transport of higher emittance particle beams but at the cost of relatively lower accelerating fields. The mode at 1.89 micron is in fact near the bottom of the band gap in Figure 4 and very near the lower allowed lattice band. Shifting this mode farther up into the band gap should improve its properties since it will be better confined. Table 1 collects various mode properties for different defect radii as obtained using the CUDOS software. The mode's wavelength shift is $\mathrm{d} \lambda / \mathrm{dR}$ $\approx-0.09$, which is very similar to the Lin accelerating mode case and may suggest a common scaling for this type of surface mode. As the defect radius increases by $2 \%$, the mode's accelerating field increases by $20 \%$ and the characteristic impedance by almost $50 \%$. The loss decreases as confinement is improved. Conversely a reduction in the radius results in the mode moving farther down into the allowed band, and its loss increases dramatically.

Table 1: Properties of SOL accelerating mode in HC1550-02 fiber for different defect radii.

\begin{tabular}{|l|l|l|l|l|}
\hline $\mathbf{R}_{\text {inner }}(\boldsymbol{\mu m})$ & $\boldsymbol{\lambda}(\boldsymbol{\mu m})$ & $\mathbf{E}_{\text {acc }} / \mathbf{E}_{\mathbf{m a x}}$ & $\mathbf{Z}_{\mathbf{C}}(\boldsymbol{\Omega})$ & $\mathbf{I m}(\mathbf{n})$ \\
\hline 5.00 & 1.8946 & 0.0493 & 0.136 & $8 \times 10^{-6}$ \\
\hline 5.10 & 1.8872 & 0.0660 & 0.250 & $1.3 \times 10^{-6}$ \\
\hline 5.20 & 1.8767 & 0.0788 & 0.371 & $10^{-6}$ \\
\hline
\end{tabular}

\section{CONCLUSION}

We are able to modify the electromagnetic properties of accelerating modes in PBG fibers by small changes in the boundary region separating the defect and surrounding matrix. Our initial study indicates that significant improvement can be made in the quality of fiber accelerator modes by this technique. Field intensities, characteristic impedances, and mode losses are very sensitive to changes in the defect geometry. This opens the possibility of active tuning of the dimensions, for example by locally controlled, thermal expansion of the lattice.

\section{REFERENCES}

[1] X.E. Lin, "Photonic band gap fiber accelerator," Phys. Rev. ST Accel. Beams 4, (2001) 051301.

[2] J.A. West et al, "Surface modes in air-core photonic band-gap fibers," Optics Express 12 (2004) 1485.

[3] M.J.F. Digonnet et al, "Understanding Air-Core Photonic-Bandgap Fibers: Analogy to Conventional Fibers," J. Lightwave Technology 23 (2005) 4169.

[4] Crystal Fibre A/S, photonic band-gap fibers online catalog, http://www.blazephotonics.com.

[5] CUDOS MOF Utilities for microstructured optical fibres, B. Kuhlmey, University. of Sydney, Australia, http://www.physics.usyd.edu.au/cudos/mofsoftware.

[6] BandSolve photonics software, from RSoft Design Group, Ossining, NY, www.rsoftdesign.com. 